Article

\title{
Taxonomic and Metabolite Diversity of Actinomycetes Associated with Three Australian Ascidians
}

\author{
Larissa Buedenbender 1,*, Anthony R. Carroll ${ }^{1}$ (D), Merrick Ekins ${ }^{2}$ (D) and D. Ípek Kurtböke ${ }^{3, *}$ \\ 1 Environmental Futures Research Institute, School of Environment, Griffith University, Gold Coast Campus, \\ Southport, QLD 4222, Australia; a.carroll@griffith.edu.au \\ 2 Biodiversity and Geosciences Program, Queensland Museum, P.O. Box 3300, South Brisbane, QLD 4101, \\ Australia; merrick.ekins@qm.qld.gov.au \\ 3 GeneCology Research Centre, Faculty of Science, Health, Education and Engineering, \\ University of the Sunshine Coast, Maroochydore, QLD 4558, Australia \\ * Correspondence: larissa.buedenbender@griffithuni.edu.au (L.B.); IKurtbok@usc.edu.au (D.İ.K.); \\ Tel.: +61-(07)-5430-2819 (D.İ.K.)
}

Received: 11 September 2017; Accepted: 15 November 2017; Published: 20 November 2017

\begin{abstract}
Actinomycetes are known to be the most prolific producers of biologically active metabolites. Here, we investigated the host species-specificity and the related secondary metabolites of actinomycetes that are associated with three different Australian ascidians, namely Symplegma rubra, Aplidium solidum, and Polyclinum vasculosum. Results indicated that while isolates from the genera Streptomyces and Micromonospora were highly diverse in the ascidian samples, only two culturable actinomycete Operational Taxonomic Units (OTUs) overlapped between all of the ascidians, pointing to some degree of host species-specificity of the isolates and selective acquisition of microbial associates by the host from the surrounding environment. LC-MS/MS profiling of extracts obtained from the ascidians and their actinomycete associates revealed many overlapping ions between hosts and actinomycetes, indicating that these compounds were likely to be synthesised by the microbial associates. Laboratory cultures of the actinomycetes displayed even more diverse metabolomes than those of their ascidian hosts; thus, making ascidian-associated actinomycetes an excellent target for natural product drug discovery and biotechnology.
\end{abstract}

Keywords: Actinobacteria; actinomycetes; Actinomycetales; marine microbial diversity; ascidian-associated actinomycetes; secondary metabolites

\section{Introduction}

Ascidians (phylum: Chordata, sub-phylum: Tunicata) are soft-bodied, sessile, filter-feeding marine invertebrates that often rely on secondary metabolites for their defence [1-3]. These compounds often show potent biological activity and thus have been recognised for their pharmaceutical and biotechnological potential. Some striking examples include ecteinascidin, marketed as Yondelis ${ }^{\circledR}$, and others undergoing clinical trials are plitidepsin and lurbinectedin [4]. Even though ascidians have immense potential for the discovery of bioactive metabolites, sustainable supply of these bioactive molecules from wild harvesting of these animals is difficult. This is because ascidians often live in large colonies of thousands of individuals, but are only a few millimetres thick.

Increasing evidence has shown that associated bacteria are the true producers of many of the ascidian's bioactive metabolites [5,6]. Therefore, isolation of microbial associates provides a sustainable source of supply for the development of novel pharmaceutical drug leads, as it permits fermentation-based production of natural products rather than the harvest of enormous amounts of 
marine invertebrate biomass $[7,8]$. The composition of the microbiome associated with the ascidian host has been shown to be species-specific, as well as influenced by the physiological environmental at the geographical location $[9,10]$. Currents, preceding weather events, and vicinity to urbanisations are some of the factors that can impact the acquisition of the microbial associates by the invertebrate host during filter feeding. These factors ought to be considered when obtaining samples in order to identify the specificity of ascidian-associated bacteria that are producers of diverse bioactive metabolites that are found in the host [11].

Even though ascidians and microorganisms are known to associate, the true nature of the relationship between the bacteria and the ascidian host remain mostly unresolved. Obligate symbiosis has been confirmed between the alpha-proteobacterium, Candidatus Endoecteinascidia faulkneri, and the ascidian Lissoclinum patella. The symbiont lives intracellularly in the blood cells of the host and is vertically transmitted over generations; as a result, the bacterium has lost its original shape and the genome has degraded to solely produce bioactive secondary metabolites [12, 13]. Symbiotic relationships have also been defined for the cyanobacterium, Prochloron didemni. However, in this case, the symbionts are found extracellularly and the bacterium has been associated with nutrition of fixed carbon and the recycling of nitrogen [14,15]. Additionally, genes that are responsible for the synthesis of highly toxic cyanobactins were identified in the P. didemni genome, but could not be found in the didemnid ascidian genome $[5,16,17]$. Chemistry of didemnid ascidians often varies between animals; therefore, an ascidian species-specific combination of horizontal and vertical transmission of symbiotic bacteria has been proposed [16,18].

Actinobacteria have been reported in marine environments and in associations with ascidians [19-21]. Current intraclass relatedness of the class Actinobacteria reveals the presence of nine orders [22]. Accordingly, "Actinobacteria" in this paper will only refer to the "actinomycetes" covering the members of the order "Actinomycetales" in the historical sense. It will also include the newly created orders Frankiales, Geodermatophiales, Kineosporiales, and Micrococcale [22], as they were part of this order until recently [23].

Actinomycetes are known as prolific producers of bioactive metabolites, and several bioactive secondary metabolites have been isolated from ascidian-associated actinomycetes [24-28]. However, most studies are inconclusive in relation to revealing any functional role of ascidian-associated actinomycetes. In most cases, the actinomycete metabolites could not be detected in their ascidian host extracts, leading to the conclusion that actinomycetes do not play an important role in secondary metabolism symbiosis and their associations to ascidians might be of facultative nature where actinomycetes are horizontally transmitted from the environment [6]. In contrast to these findings, several staurosporine derivatives, a structure class known to be synthesised by terrestrial and marine actinomycetes, have repeatedly been isolated from Eudistoma ascidians [29], pointing towards a functional role of staurosporine production by a symbiotic actinomycetes strain [30].

The Polyclinidae and Styelidae are two of three ascidian families from which the majority of bioactive natural products have so far been isolated [31], many of which are likely to be of microbial origin [31]. Yet, few attempts have been made to investigate their microbial associates. Herein, we selected Aplidium solidum and Polyclinum vasculosum, both belonging to the same taxonomic family, Polyclinidae, which are abundant species at Hastings Point in Northern New South Wales, Australia, as well as Symplegma rubra (Styelidae) from the same marine environment to explore species-specificity of their associated actinomycete diversity that had not been previously examined. As it is suggested that actinomycetes are horizontally acquired from the environment, we hypothesised that consequently their diversity should greatly overlap between the three-different species that are sampled at the same location. To evaluate this hypothesis, we initially examined the overall microbial diversity that is associated with the three Australian ascidians to identify the presence of actinomycetes based on a culture-independent approach. We then focused our culturing efforts to isolate actinomycetes and conducted molecular networking of actinomycete extracts, as well as ascidian extracts, to depict 
their metabolic diversity and identify metabolites of the ascidian metabolome that are potentially synthesised by an actinomycete associate.

\section{Materials and Methods}

\subsection{Site Description and Ascidian Collection}

Hastings Point is a rocky shore habitat that receives warm waters from the East Australian Current containing tropical marine invertebrate larvae as well as more temperate waters cycling back from the South [32]. The intermixing of cold and warm waters results in a rich and unique biodiversity of marine invertebrates, predominantly ascidians, making the location a prime target for biodiscovery. For this study, three different ascidian specimens were collected. Aplidium solidum (QM G335842) and Polyclinum vasculosum (QM G335843), both belonging to the same taxonomic family, Polyclinidae, were obtained on 13 July 2015 from rock pools at depths between 1-2 m at Hastings Point, New South Wales, Australia ( $28^{\circ} 22^{\prime} \mathrm{S}, 153^{\circ} 35^{\prime}$ E). Symplegma rubra (QM G335841), family Styelidae, was collected by snorkel at a depth of $6 \mathrm{~m}$ at a rocky reef approximately $200 \mathrm{~m}$ offshore from Hastings Point headland (Site 1, Figure 1a).

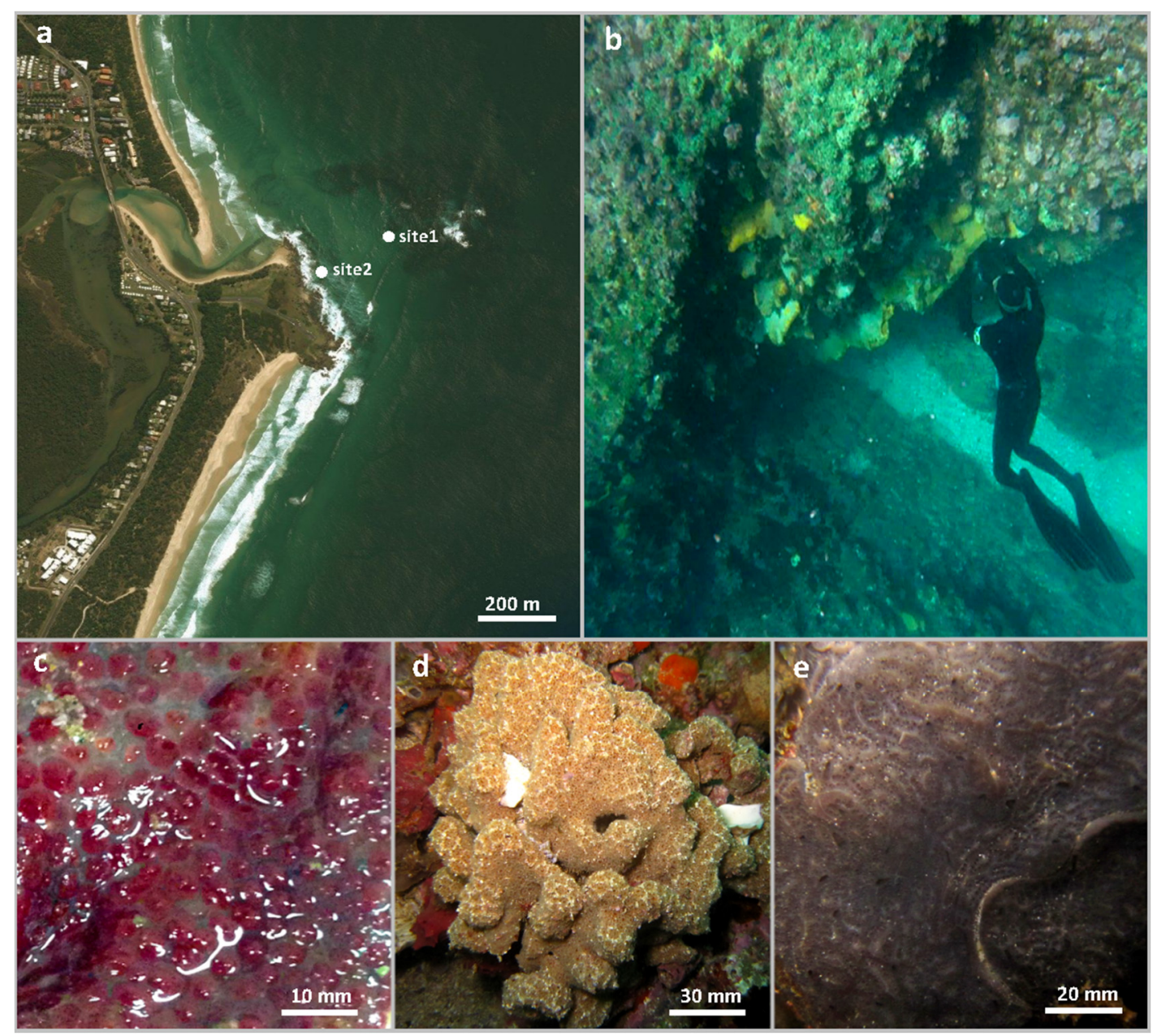

Figure 1. The Northern New South Wales coastline and in-situ aspects of the Hastings Point rock pools (a) Location of Hastings Point along the Northern New South Wales coastline and sampling sites [33];

(b) Topography of the rocky reef at site 1; (c) S. rubra; (d) A. solidum; and, (e) P. vasculosum.

All three ascidians are colonial species with microsize tunics (approximately 1-4 mm thick). The samples were collected at low tide under a Scientific Collection Permit (Permit No: P09/0031-3.0) 
that was obtained from the New South Wales Department of Primary Industries. Ascidian samples were placed into separate plastic zip lock bags containing seawater and were transported to the laboratory on ice within $2 \mathrm{~h}$ of sampling. About $1-16 \mathrm{~g}$ of ascidian tissue was exhaustively extracted with methanol to obtain a crude chemical profile of each specimen. Preserved ascidian specimens were deposited at the Queensland Museum, Brisbane where they were identified taxonomically at the species level.

\subsection{Culture-Independent Microbial Diversity Analysis}

Three $1 \mathrm{~mm}^{3}$ cubes were cut out from different locations of each ascidian specimen to allow for a representative sample of the whole organism. The tissue sections were washed with sterile seawater and microbial DNA was extracted with the FastDNA SPIN Kit for Soil (MP Biomedicals). The extracted DNA was sent to the Australian Genome Research Facility (AGRF) for microbial diversity Illumina sequencing. The DNA samples were amplified using the primers 341F (CCTAYGGGRBGCASCAG) and 806R (GGACTACNNGGGTATCTAAT) for the V3 and V4 regions of the 16S rRNA gene. Forward and reverse microbiome sequences were quality filtered with Trimmomatic [34] and were joined using PandaSeq [35]. In the software package Quantitative Insights Into Microbial Ecology (QIIME) version 1.9.1 [36], the joined sequences of all three ascidian microbiomes were combined to one fasta file using the command add_qiime_labels.py. The workflow script pick_de_novo_otus.py was used to pick de novo OTUs (Operational Taxonomic Units) based on 97\% sequence similarity; one representative sequence for each OTU was selected and used to assign taxonomy; subsequently, OTU sequences were aligned to build a phylogenetic tree. The summarize_taxa_through_plots.py script was run to group OTUs to phylum, class and family levels; these tables were exported to Microsoft Excel to illustrate the phylum assignments as a bar chart. Alpha rarefaction plots were generated through alpha_rare_faction.py. The sequences of OTUs belonging to actinomycetes were individually searched against the BLASTn database to confirm family level assignments.

\subsection{Culture-Dependent Actinomycete Isolation and Identification}

The ascidian specimens were first subjected to surface sterilization by rinsing five times with sterile seawater to remove transient and loosely attached bacteria. Each ascidian was cut into small pieces of $5 \mathrm{~mm}^{3}$ that were briefly rinsed with $70 \% \mathrm{EtOH}$, and then again rinsed with sterile seawater. The ascidian tissue was homogenised with a sterile mortar and pestle with $10 \mathrm{~mL}$ of sterile seawater; the extract and ascidian tissue were transferred to a $15 \mathrm{~mL}$ Eppendorf tube and vortexed for $10 \mathrm{~min}$. The supernatants were then diluted (1:2 and 1:20) and $250 \mu \mathrm{L}$ were plated in duplicates on agar plates. Five different microbiological media; Difco marine agar 2216 (Difco Laboratories, Detroit, MI, USA), Starch Casein Agar (SCA; $10.0 \mathrm{~g}$ starch, $2.0 \mathrm{~g} \mathrm{~K}_{2} \mathrm{HPO}_{4}, 2.0 \mathrm{~g} \mathrm{HNO}_{3}, 2.0 \mathrm{~g} \mathrm{NaCl}, 0.3 \mathrm{~g}$ casein, $0.05 \mathrm{~g} \mathrm{MgSO}_{4} \cdot 7 \mathrm{H}_{2} \mathrm{O}, 20.0 \mathrm{~g}$ agar [37]), Starch Agar (SA; 10.0 g glucose, 10.0 g potato starch, $10.0 \mathrm{~g}$ corn starch, $3.0 \mathrm{~g}$ yeast extract, $4.0 \mathrm{~g}$ peptone, $4.0 \mathrm{~g}$ beef extract, $33.3 \mathrm{~g}$ artificial sea salt, 20.0 g agar [38]), Corn Starch (CS; $20.0 \mathrm{~g}$ corn starch, $10.0 \mathrm{~g}$ glucose, $10.0 \mathrm{~g}$ peptone, 6.0 g yeast extract, agar $20.0 \mathrm{~g}$ [38]), Nocardia histidans medium (NH; $10.0 \mathrm{~g}$ yeast extract, $10.0 \mathrm{~g}$ glucose, $0.95 \mathrm{~g} \mathrm{Na}_{2} \mathrm{HPO}_{4}$, $0.91 \mathrm{~g} \mathrm{KH}_{2} \mathrm{PO}_{4}, 0.5 \mathrm{~g} \mathrm{MgSO} \cdot 7 \mathrm{H}_{2} \mathrm{O}, 20.0 \mathrm{~g}$ agar [39]); were used for the isolation of actinomycetes. All media were supplemented with $50 \mu \mathrm{g} / \mathrm{mL}$ cycloheximide and $50 \mu \mathrm{g} / \mathrm{mL}$ nystatin to prevent fungal growth, as well as $25 \mu \mathrm{g} / \mathrm{mL}$ nalidixic acid to inhibit the growth of background non-actinomycete bacteria. After 8 days of incubation at $28^{\circ} \mathrm{C}$, actinomycete colonies were picked from the plates and were re-streaked on fresh Oatmeal Agar plates (OMA; $20 \mathrm{~g}$ oatmeal, $20 \mathrm{~g}$ bacteriological agar, $3 \mathrm{~g}$ yeast extract [40]) for purification and morphological investigation; this was repeated until pure cultures were obtained. The pure cultures of actinomycetes were given a unique numerical registration number at the Genecology Research Centre at the University of the Sunshine Coast, with a code prefix of "USC". For long term storage actinomycete isolates were kept in $20 \%$ glycerol at $-20^{\circ} \mathrm{C}$ and $-80^{\circ} \mathrm{C}$ [41]. 


\subsection{Molecular Identification and Phylogenetic Analysis of Actinomycete Isolates}

Actinomycete DNA was extracted from individual colonies using the DNeasy Blood and Tissue Kit (Qiagen, Hilden, Germany), as per the manufacturer's instructions for gram-positive microorganisms. PCR was performed to amplify the $16 \mathrm{~S}$ rRNA gene, therefore, $4 \mu \mathrm{L}$ of DNA extract were used as template in $40 \mu \mathrm{L}$ solution containing $1 \mathrm{mM}$ of forward primer 27F (AGAGTTTGATCMTGGCTCAG) and $1 \mathrm{mM}$ of reverse 1492R (TACGGYTACCTTGTTACGACTT), $20 \mu \mathrm{L}$ of TopTaq Master Mix (Qiagen, Germany), and $14 \mu \mathrm{L}$ of sterile $\mathrm{H}_{2} \mathrm{O}$. Amplification was performed on a Thermocycler 100 (BioRad, Hercules, CA, USA) under following conditions: initial denaturation (10 $\mathrm{min}$ at $95^{\circ} \mathrm{C}$ ); followed by 25 cycles of denaturation $\left(10 \mathrm{~s}\right.$ at $\left.95^{\circ} \mathrm{C}\right)$, primer annealing $\left(15 \mathrm{~s}\right.$ at $\left.55^{\circ} \mathrm{C}\right)$, primer extension $\left(1: 20 \mathrm{~min}\right.$ at $\left.72{ }^{\circ} \mathrm{C}\right)$; and, final extension $\left(10 \mathrm{~min}\right.$ at $\left.72{ }^{\circ} \mathrm{C}\right)$. To test whether the amplification was successful, gel electrophoresis was conducted at $100 \mathrm{~V}$ for $45 \mathrm{~min}$ on $1.2 \%$ agarose gel in $0.6 \% \mathrm{TAE}$ buffer stained with ethidium bromide. The presence of the $16 \mathrm{~S}$ rRNA fragment was inspected under UV light using a Bio-Rad ChemiDoc XRS+ imaging system. Sequencing was performed by Macrogen Inc., Seoul, Korea (http://macrogen.com/eng/). Actinomycete DNA sequences were pairwise aligned and trimmed in Geneious R6.1.8; the data was then searched against the National Center for Biotechnology Information (NCBI) nucleotide database (http:/ / www.ncbi.nlm.nih.gov/BLAST/) to find the closest relative based on \%-identity and query cover scores. All associate sequences were multiple aligned using MUSCLE (Multiple Sequence Comparison by Log-Expectation [42]). The best evolutionary model was determined based on the lowest BIC (Bayesian Information Criterion) and AIC (Akaike Information Criterion) values. A Maximum Likelihood phylogeny was constructed in MEGA 7.0.21 using the model GTR+I+G. The 16S rRNA sequence for Bifidobacterium angulatum was downloaded from NCBI and used as an outgroup. The robustness of the phylogenetic tree was evaluated by bootstrap analysis with 1000 re-samplings. Furthermore, the sequence data was imported into QIIME, de novo OTUs were established using pick_de_novo_otus.py based on 99.9\% sequence similarity.

\subsection{Metabolite Diversity}

The 120 actinomycete isolates were incubated in Corning ${ }^{\circledR}$ (Corning, NY, USA) MiniBioreactors with $30 \mathrm{~mL}$ ASW-A (20.0 g soluble starch, $10.0 \mathrm{~g}$ glucose, $5.0 \mathrm{~g}$ peptone, $5.0 \mathrm{~g}$ yeast extract, $5.0 \mathrm{~g} \mathrm{CaCO}_{3}$ per litre of artificial seawater) at $28^{\circ} \mathrm{C}$ and $250 \mathrm{rpm}$. After two weeks, the tubes were centrifuged at $8000 \mathrm{rpm}$ for $10 \mathrm{~min}$, and the liquid media supernatant was collected. Cells were washed with $15 \mathrm{~mL}$ chilled deionised water, vortexed, and centrifuged at $8000 \mathrm{rpm}$, and again supernatant was collected. Methanol $(15 \mathrm{~mL})$ was added to the harvested microbial cell pellet and the mixture was incubated on a rotational shaker for $2 \mathrm{~h}$ at room temperature and $200 \mathrm{rpm}$. The liquid phase was separated by centrifugation ( $8000 \mathrm{rpm}, 5 \mathrm{~min}$ ) and subsequently collected. The methanol extraction of the cells was repeated with rotation overnight. The combined extracts were filtered using a $0.22 \mu \mathrm{m}$-pore-size filter and dried under pressure (GeneVac Technologies HP-12). Methanol was chosen as the extraction solvent due to its amphiphilic characteristics that enable the extraction of a very broad range of polar and non-polar metabolites.

Microbial and ascidian crude extracts were prepared at $2 \mathrm{mg} / \mathrm{mL}$ and analysed on a 6530 Accurate Mass Q-TOF spectrometer (Agilent Technologies, Santa Clara, CA, USA) workstation coupled to an Agilent $1260 \mathrm{LC}$ system using positive electrospray ionization. A Kinetex ${ }^{\circledR} \mathrm{C}_{18} 5 \mu \mathrm{m} 100 \AA \mathrm{A} \mathrm{HPLC}$ column $(100 \times 4.6 \mathrm{~mm})$ was used under chromatographic conditions from $0-100 \% \mathrm{ACN} / 0.1 \% \mathrm{FA}$ over $20 \mathrm{~min}$. The Q-TOF MS was set to positive mode mass range $m / z$ 100-1700, MS scan rate 3 spectra/s, MS/MS scan rate 2/s, fixed collision energies at 10, 20, and $40 \mathrm{keV}$. Source parameters were set to gas temperature $300{ }^{\circ} \mathrm{C}$, gas flow $10 \mathrm{~L} / \mathrm{min}$, nebulizer $35 \mathrm{psig}$; and, scan source parameters were: VCap 3500, nozzle voltage $1500 \mathrm{~V}$, fragmentor $130 \mathrm{~V}$, skimmer $65 \mathrm{~V}$, and octopoleRFPeak $750 \mathrm{~V}$.

Every fifth acquired sample was followed by a blank. At the beginning and end of the LC-MS/MS acquisition, a $10 \mu \mathrm{g} / \mathrm{mL}$ mixed pesticide standard (acidic and basic, Agilent Technologies) was tested as a quality control. Data was inspected for integrity in MassHunter software (Agilent Technologies). 
For data analysis, all MS/MS data was converted from Agilent MassHunter data files to mzXML file format using MSConvert. The data was uploaded to the GNPS-Global Natural Products Social molecular networking server (gnps.ucsd.edu). A molecular network was created using the online workflow at GNPS. The data was filtered by removing all of the MS/MS peaks within $\pm 17 \mathrm{Da}$ of the precursor $m / z$. MS/MS spectra were window filtered by choosing only the top six peaks in the $\pm 50 \mathrm{Da}$ window throughout the spectrum. The data was then clustered with MS-Cluster with a parent mass tolerance of 2.0 Da and a MS/MS fragment ion tolerance of 0.5 Da to create consensus spectra. Consensus spectra that contained less than two spectra were discarded. A network was then created where edges were filtered to have a cosine score above 0.7 and more than four matched peaks. Further edges between two nodes were kept in the network only if each of the nodes appeared in each other's respective top 10 most similar nodes. The spectra in the network were then searched against GNPS spectral libraries. The library spectra were filtered in the same manner as the input data. All of the matches kept between network spectra and library spectra were required to have a score above 0.7 and at least four matched peaks [43]. The molecular network was visualised in Cytoscape 3.3.0., where all of the mass ions that were associated with the blanks and mixed pesticide standard were removed from the network. Nodes were coloured per actinomycete taxa group or ascidian group. The network figure was edited in Inkscape 0.92 freeware.

Euler-Venn diagrams were generated with eulerAPE v3 (http://www.eulerdiagrams.org/ eulerAPE) to depict the numbers of unique and shared actinomycete OTUs between the three ascidians based on the data that was obtained through culture-independent and culture-dependent approaches. Similarly, shared secondary metabolite mass ions of the three ascidians, as well as their cultured ascidian-associated actinomycetes, were analysed. Thereby, any primary metabolites that were identified against the GNPS database were excluded from the Euler-Venn diagrams.

\section{Results}

\subsection{Microbial Diversity Associated with Three Australian Ascidians}

We evaluated the microbial diversity of Australian ascidians using a culture-independent approach targeting ascidian genera, whose microbiomes had not previously been studied in breadth. The samples were collected at an intertidal habitat at Hastings Point, New South Wales, Australia and based on morphological traits the specimens were taxonomically assigned to Symplegma rubra (Figure 1c), Aplidium solidum (Figure 1d), and Polyclinum vasculosum (Figure 1e).

16S rRNA gene Illumina sequencing of the V3-V4 region resulted in a total of 287,758 sequences for the three ascidians. After quality filtering, 253,962 sequences remained for analysis and 13,945 OTUs were determined based on $97 \%$ sequence similarity. Species richness was established through alpha rarefaction and was compared between the three ascidian samples. From the rarefaction curves (Figure 2a) it was apparent that $S$. rubra, which was the only sample collected at the small rocky reef approximately $100 \mathrm{~m}$ offshore, had a more diverse microbial community than the other two ascidian species, P. vasculosum and A. solidum, which were obtained from sheltered intertidal rock pools.

Nineteen different bacterial phyla were observed and all three ascidian samples were dominated by Proteobacteria (54-58\%), followed by Cyanobacteria, which showed a larger variation between $11-35 \%$. All of the ascidians contained a fraction of unassigned phyla; specifically, in P. vasculosum, this fraction had a high contribution of $28 \%$ of the overall bacterial diversity. The phylum Actinobacteria was present in all three ascidians; however, in a far smaller portion, with the highest percent observed in S. rubra being $1.91 \%$, while A. solidum and P. vasculosum both had values below $1 \%$ (Figure $2 \mathrm{~b}$ ). Within the phylum Actinobacteria, the order Actinomycetales is of particular interest due to their ability to produce many bioactive metabolites. Therefore, we further inspected the order Actinomycetales to the family level (Table 1). Sequences of the taxonomic families Corynebacteriaceae, Microbacteriaceae, Nocaridaceae, and Sterptomycetaceae were detected in all three ascidians, yet at the OTU level (97\%) 
only two OTUs were shared between all three ascidians (Table 1 and Figure 3a). Thermomonosporaceae and Mycobacteriaceae had the highest number of OTUs, but sequences were only detected in S. rubra and P. vasculosum. In these two ascidians, the natural product producing families Micromonosporaceae and Streptosporangiaceae were discovered, as well as Kineosporiaceae. A. solidum exhibited the lowest diversity of actinomycete families; while Actinomycetaceae and Micrococcaceae were unique to this ascidian, Pseudonocardiaceae OTUs were shared with S. rubra. Four OTUs that could not be identified to family level unambiguously were obtained from A. solidum and P. vasculosum. All of the other observed actinomycetes family OTUs were unique to only one ascidian (Table 1).
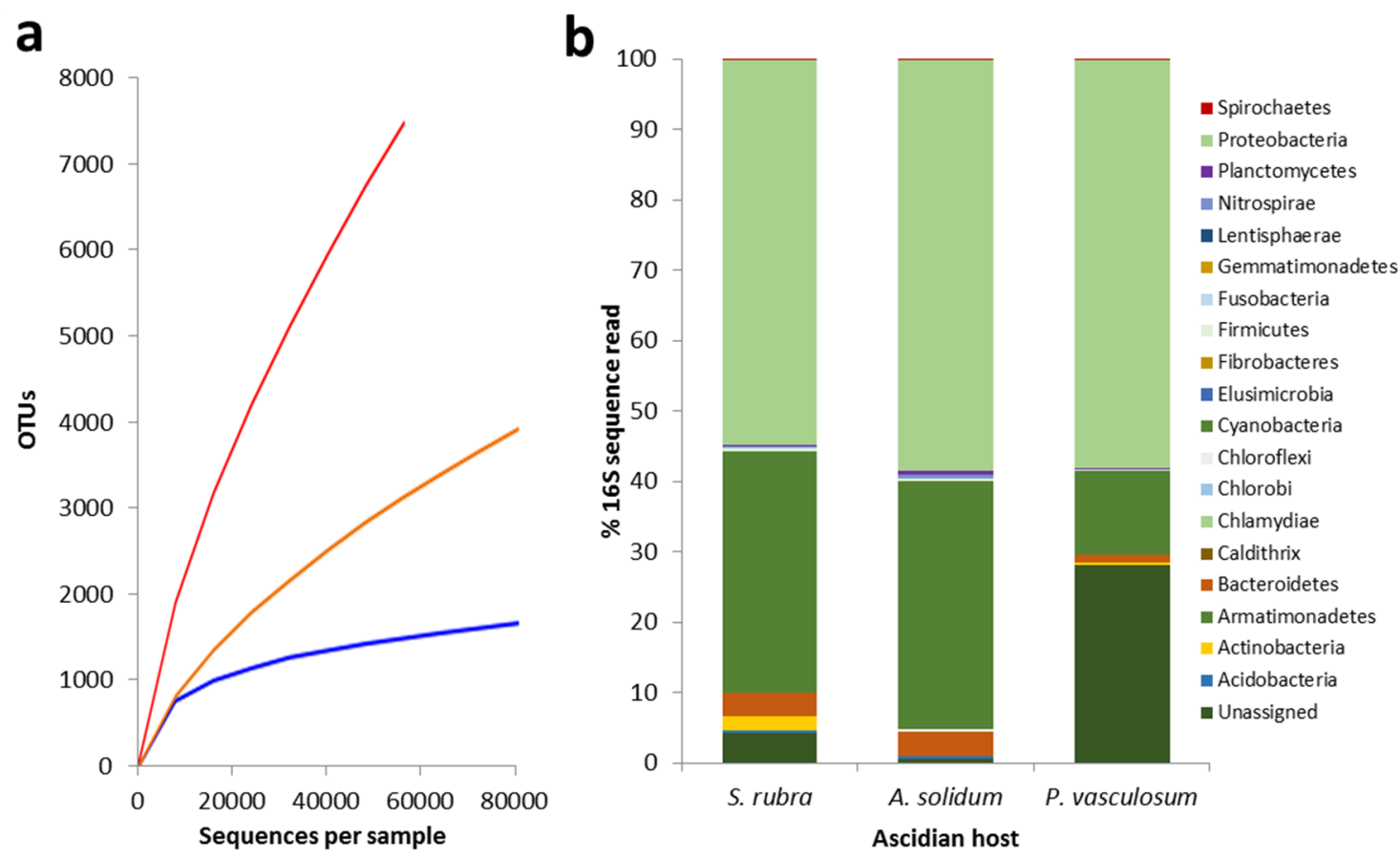

Figure 2. Microbial diversity analysis (a) Average alpha rarefaction curve showing that $S$. rubra (red) contained the highest microbial diversity, while A. solidum (blue) had the least diverse microbial community; (b) Percent diversity of taxonomic phyla assigned to three Australian ascidian species.

Table 1. Summary of de novo Operational Taxonomic Units (OTUs) representing the taxonomic order "Actinomycetales".

\begin{tabular}{ccc}
\hline No of OTUs & Family & Ascidian Host \\
\hline 1 & Acidothermaceae & Polyclinum vasculosum \\
1 & Actinomycetaceae & Aplidium solidum \\
1 & Cellulomonadaceae & Polyclinum vasculosum \\
2 & Corynebacteriaceae & Symplegma rubra, Polyclinum vasculosum, Aplidium solidum \\
1 & Gordoniaceae & Symplegma rubra \\
2 & Intrasporangiaceae & Polyclinum vasculosum \\
1 & Kineosporiaceae & Symplegma rubra, Polyclinum vasculosum \\
5 & Microbacteriaceae & Symplegma rubra, Polyclinum vasculosum, Aplidium solidum \\
1 & Micrococcaceae & Aplidium solidum \\
3 & Micromonosporaceae & Symplegma rubra, Polyclinum vasculosum \\
6 & Mycobacteriaceae & Symplegma rubra, Polyclinum vasculosum \\
5 & Nocaridaceae & Polyclinum vasculosum \\
1 & Promicromonosporaceae & Symplidium solidum \\
1 & Pseudonocardiaceae & Sympra, Polyclinum vasculosum , Aplidium solidum \\
4 & Streptomycetaceae & Symplegma rubra, Polyclinum vasculosum, Aplidium solidum \\
3 & Streptosporangiaceae & Symplegma rubra, Polyclinum vasculosum \\
6 & Thermomonosporaceae & Symplegma rubra, Polyclinum vasculosum \\
4 & Ambiguous assignment & Symplegma rubra, Polyclinum vasculosum \\
\hline
\end{tabular}


a

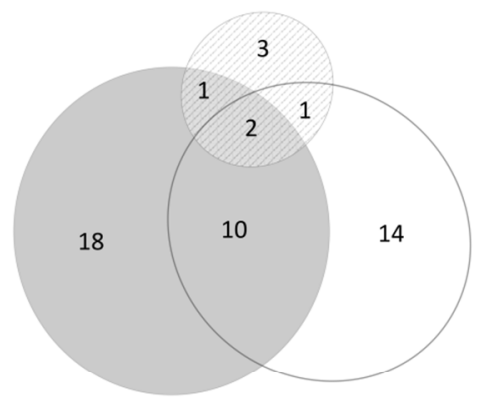

Detectable ascidian-associate actinomycete OTUs

C

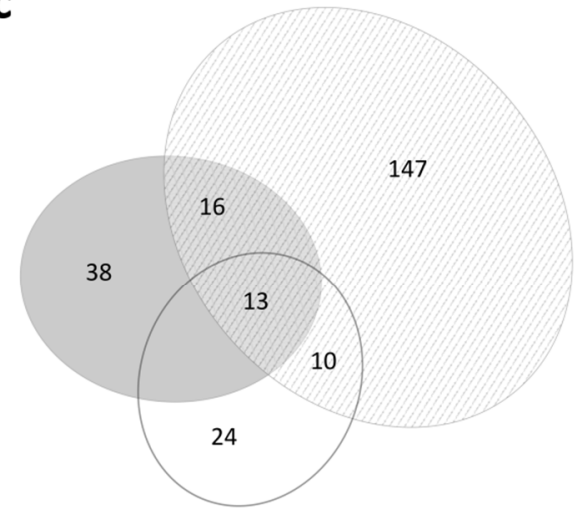

Ascidian metabolites

Symplegma rubra b

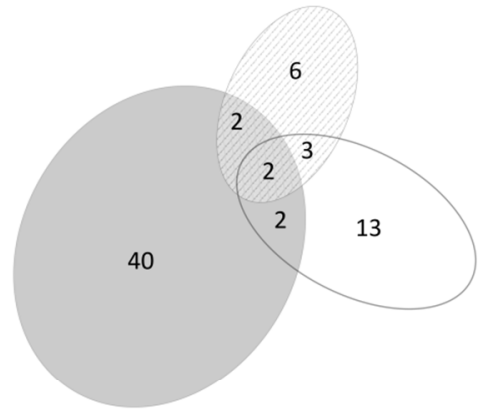

Culturable ascidian-associate actinomycete OTUs

d

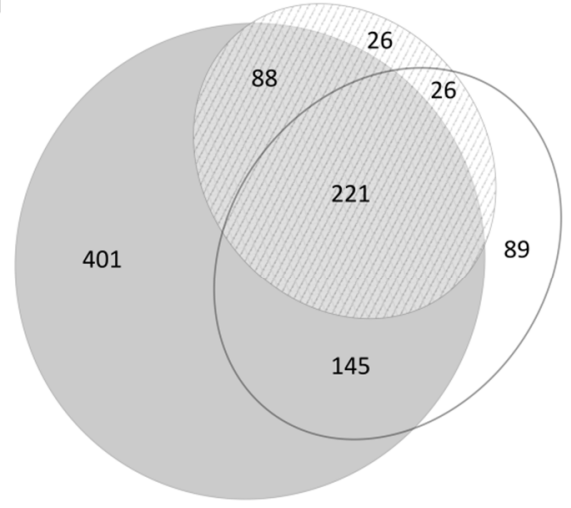

Ascidian-associate actinomycete metabolites

Figure 3. Microbial and secondary metabolite overlaps between the three Australian ascidians in terms of (a) detectable actinomycete OTUs (b) culturable actinomycete OTUs (c) molecular ions (d) associate metabolites. (Note: primary metabolite database hits were excluded from this diagram).

\subsection{Culture-Dependent Actinomycete Diversity}

Once the presence of actinomycete sequences in the different ascidians was confirmed through culture-independent techniques, traditional culture-dependent microbiological techniques were used to obtain the culturable actinomycete diversity. This resulted in the isolation of 120 diverse and morphologically actinomycete resembling isolates. Phylogenetic analysis was subsequently used to confirm their true taxonomic identitiesy. Therefore, the 16S rRNA gene was amplified for all culturable actinomycete-like isolates and subsequently sequenced. All of the sequences were amplified successfully for more than $1200 \mathrm{bp}$ using the $27 \mathrm{~F}$ and 1492R universal primers. The 120 sequences were searched against the NCBI $16 \mathrm{~S}$ ribosomal RNA database for bacteria and archaea and sequences of their closest phylogenetic relative were obtained from the database and were included in the phylogenetic analysis. One hundred and eighteen strains exhibited $99 \%$ and above similarities as compared to previously described actinomycete strains. Lower sequence similarities were observed for Streptomyces isolates USC-16002 (98\%) and USC-16062 (97\%), both isolated from the S. rubra, suggesting that these are potential candidates of novel actinomycete taxa.

The maximum likelihood analysis was used to estimate the phylogenetic relationship of the ascidian-associated actinomycete isolates and revealed four major clades with high statistical support (bootstrap values of $98-100 \%$ ). This included a clade for Micromonospaceae, Streptosporangaceae, Streptomyceae, and a Nocardiacea clade that further branched into well-supported Nocardia and Rhodococcus genus clades (Figure 4). 


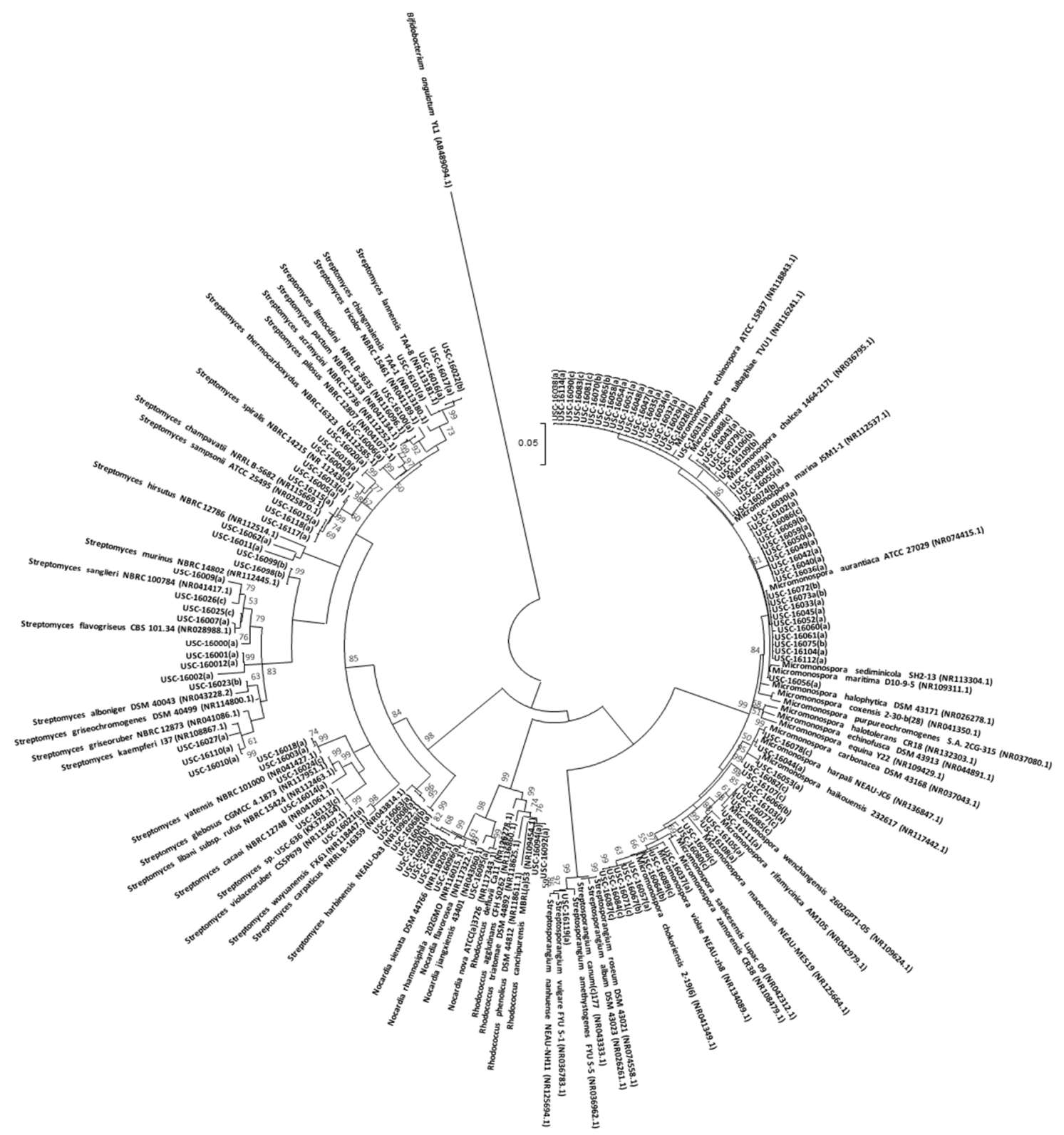

Figure 4. Phylogenetic analysis of the actinomycete isolates in relation to their closest relatives. (Bifidobacterium angulatum (AB489094) was used as an out-group sequence to root the tree). The ascidian hosts of the bacterial isolates are indicated by suffix of the isolate USC code (a) for S. rubra, (b) for A. solidum and (c) for P. vasculosum.

The three ascidian specimens yielded a total of 120 actinomycete isolates originating from five different genera: Micromonospora (58\%), Streptomyces (34\%), Nocardia (5\%), Rhodococcus ( $2 \%)$, and Streptosporangium (1\%) (Table S1). Four actinomycete-specific isolation media and one generic commercially available marine agar were chosen for isolation. The selection of media had a notable influence on the number of isolates recovered. Starch Casein Agar (SCA) allowed for the highest recovery of isolates and a total of 60 actinomycetes were obtained, predominantly from the genus Micromonospora. The highest actinomycete diversity was recovered from Corn Starch (CS) media; however, it appeared as the most selective for Streptomyces species. Starch Agar (SA) was highly selective for Micromonospora species and yielded 20 Micromonospora isolates and only one Streptomyces species. NH media only recovered one Nocardia sp. isolate. A low number of actinomycetes (6 isolates) 
were obtained from Marine Agar (MA); but interestingly, the only Streptosporangium isolate (USC-16119) was recovered from this media (Figure 5a).
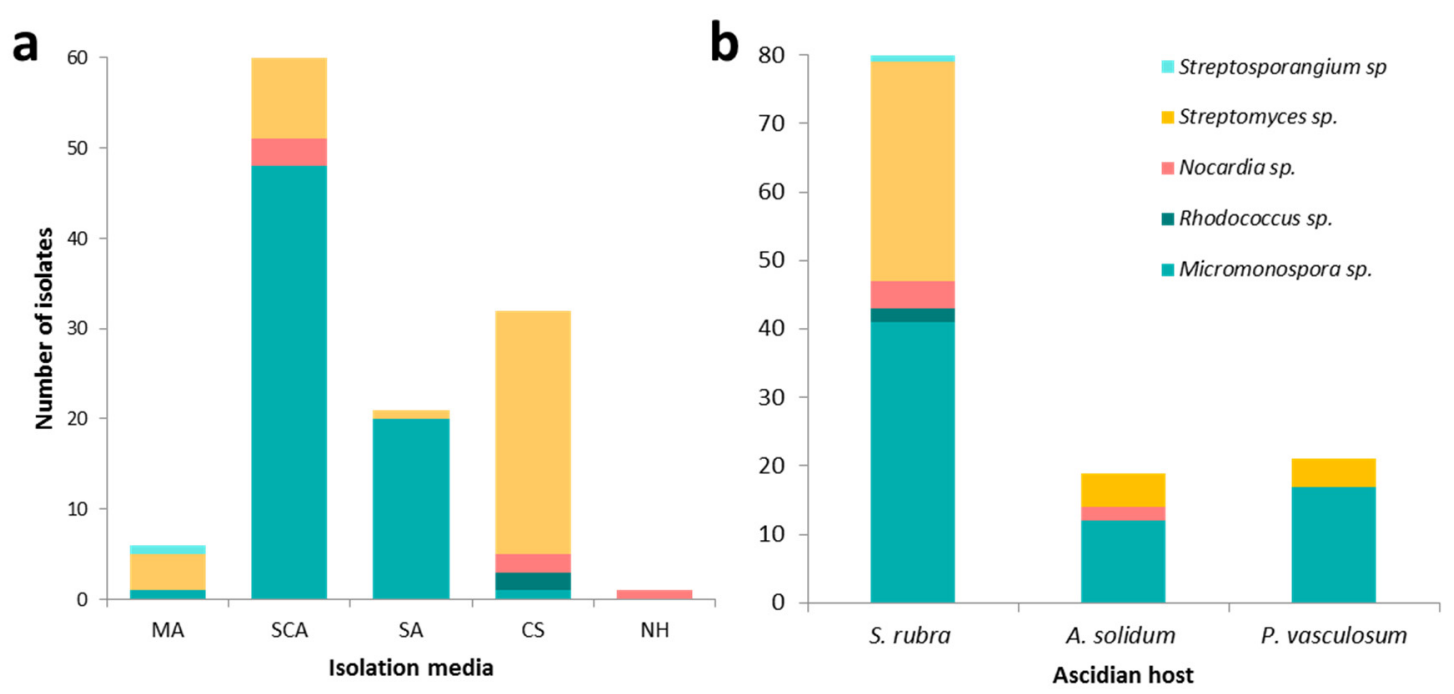

Figure 5. Numbers and taxonomic diversity of actinomycetes (a) isolated from five different isolation media; (b) derived from three Australian Ascidians.

The recovery of actinomycetes varied between the three ascidian samples. S. rubra yielded the highest number (80) and diversity of isolates with representatives of all five isolated actinomycete genera, Micromonospora, Streptomyces, Nocardia, Rhodococcus, and Streptosporangium. A. solidum and P. vasculosum, which were both collected from the shore rock pools, yielded a similar number of isolates, 19 and 21, respectively, but much lower numbers and diversity in comparison to $S$. rubra, which was collected from the rocky reef $100 \mathrm{~m}$ offshore (Figure 1a). The isolation success, therefore, reflects the observed actinomycete distribution detected through molecular analysis.

As indicated in the phylogenetic tree, several of the isolated actinomycetes appeared to be identical; therefore, the QIIME toolbox was utilised to determine culturable actinomycete OTUs, which were compared between three ascidian samples. The 120 actinomycete sequences clustered into a total of 78 OTUs based on $99.9 \%$ sequence similarity (Table S1). Figure 3 depicts the microbial and metabolite overlaps between the three Australian ascidians. Interestingly, only two microbial OTUs were shared between all of the tree ascidian specimens. This suggests that the isolated actinomycete associates were mostly unique to their ascidian host. Specifically, the Streptomyces, except for one OTU (comprised for one isolate from S. rubra and one from A. solidum), showed no overlap between ascidian samples (Table S1). The numbers of detectable and culturable actinomycetes OTUs shared between the ascidians appeared mostly proportional, only shared OTUs between S. rubra and $P$. vasculosum were higher with ten shared OTUs as compared to the two cultured OTUs (Figure 3a,b). Interestingly, the two actinomycete OTUs shared between all three ascidians that were defined based on the culture-independent methods belonged to the families of Streptomycetaceae and Nocardiaceae. We compared the sequences from culture-independent and dependent approaches and found that they well aligned, but none of the sequences matched $100 \%$. However, $16 \mathrm{~S}$ sequence fragments in the culture independent analysis were maximal $465 \mathrm{bp}$ long and of lower quality, while sequences of $1200 \mathrm{bp}$ were recovered from the cultured isolates, making definite conclusions about shared OTUs difficult.

\subsection{Metabolite Diversity}

LC-MS/MS data was collected for the three ascidian hosts, as well as extracts of the actinomycete associates. In contrast to the microbial diversity, $A$. solidum had the highest secondary metabolite diversity (186 molecular ions), followed by S. rubra (73 ions) and P. vasculosum had the lowest ion diversity (55 ions). Thirteen molecular ions were found in all three ascidian specimens (Figure 3c). 
The metabolite diversity of the ascidian-associated actinomycetes reflected the diversity of the microbial diversity and S. rubra-associated actinomycetes by far had the highest abundance of molecular ions (Figure 3d). For visualisation, a molecular ion network was constructed (Figure 6). After the removal of ions that were present in blanks and standards, a total of 1532 parent ions were observed in the samples and represented as nodes coloured by taxa where edges indicate significant pairwise alignment between nodes. The network shows several taxa-specific node clusters; specifically, Streptomyces and Micromonospora display a diverse plethora of unique molecular ion clusters. With 842 detected ions, Streptomyces exhibited the richest metabolite diversity, followed by 681 ions that were detected in Micromonospora isolates. Nocardia showed several unique clusters but had most ion overlap with Micromonospora. Only one Streptosporangium isolate was included in the analysis, yet its metabolite diversity is noteworthy with a total of 91 molecular ions detected, of which 20 were unique and 73 coincided with the Micromonospora ions. Seventy-four metabolites could be identified through MS/MS spectra matches against the GNPS database (Table S2). The majority of the database matches present common primary metabolites, such as nucleotides, sugars, amino acids, fatty acids, vitamins, and cell membrane components (phosphatidylcholines), which are of less interest for biotechnological or pharmaceutical studies. Furthermore, several linear and cyclic dipeptides, as well as other previously described bioactive secondary metabolites were identified (Figure 6a). Surugamide A and leupeptin, both protease inhibitors that were previously isolated from Streptomyces sp. [44,45], which could be annotated to Streptomyces specific clusters (Figure 6 b,c). Rakicidin A and B were attributed to a small cluster of only three nodes that were shared between several Micromonospora and the Streptosporangium isolates (Figure 6d); these compounds were previously reported to exhibit cytotoxic and anti-tumor properties [46]. Additionally, the common actinomycete siderophore for iron acquisition, desferrioxamine B, was linked to a shared actinomycete cluster (Figure 6e). The remaining vast majority of ions in the networks could not be assigned against the GNPS database.

Of great interest were the nodes that overlapped between ascidian hosts and their microbial associates, as these nodes are likely to be compounds that are produced by a microbial associate of the ascidians. Only one detected ion with a molecular mass of 479.9 Da was identified as an oxobutanoic acid derivative and was found in all three ascidians and Micromonospora and Nocardia associates. Ninety-one molecular ions coincided between S. rubra and its actinomycete associates. Whilst $P$. vasulosum had 74 overlapping ions, and in A. solidum 52 ions matched the associates. Micromonospora and Streptomyces isolate metabolomes showed the greatest overlap and thus appear to contribute to the ascidian metabolome. 


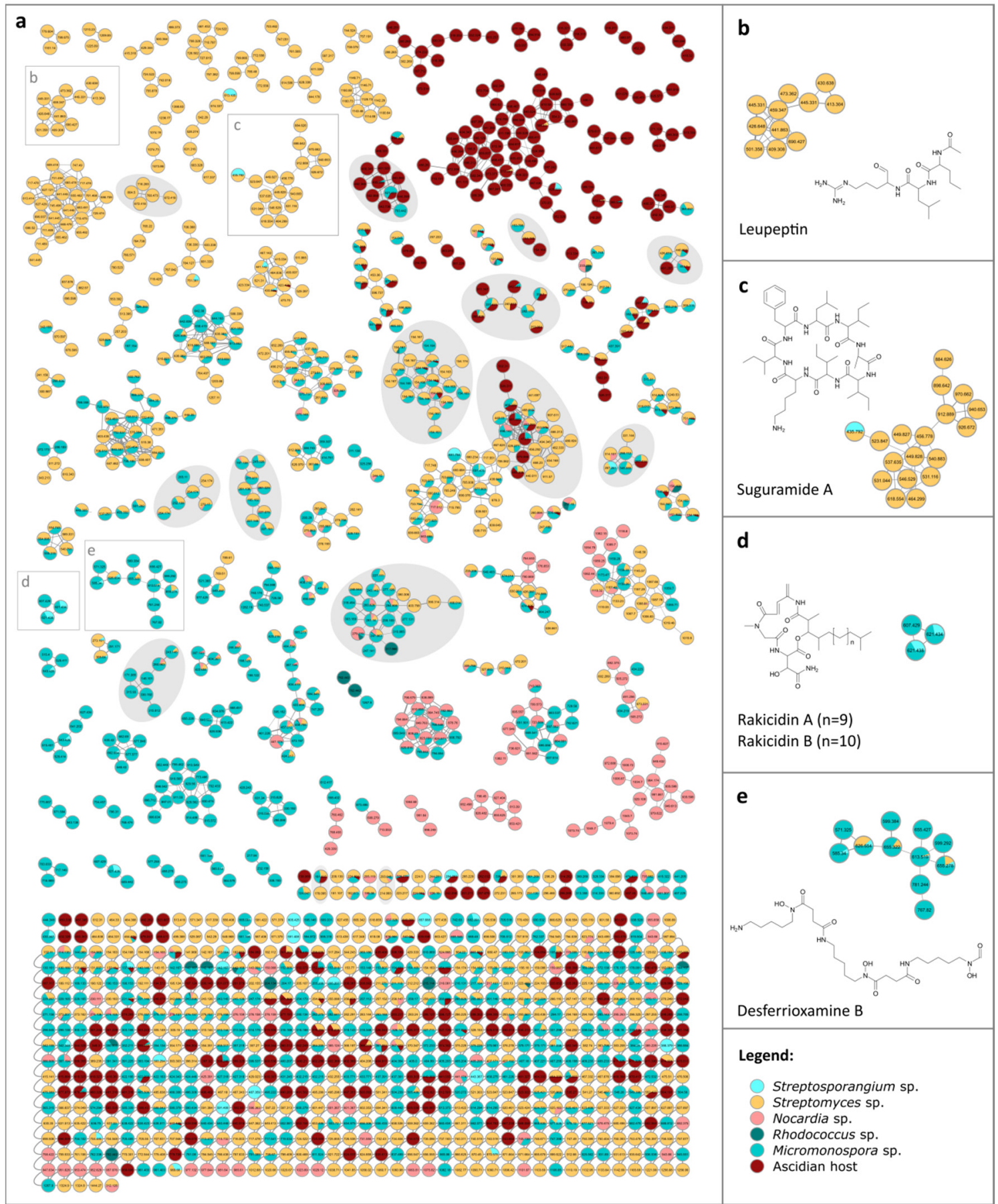

Figure 6. Ascidian-associated actinomycete molecular network: (a) overall molecular network, where node colour represents different actinomycete taxa and ascidian hosts; networks with identified primary metabolites are highlighted with grey ellipses. Identified secondary metabolite networks of (b) leupeptin; (c) surugamide A; (d) rakicidin A and B; (e) desferrioxamine B.

\section{Discussion}

In this study, we compared the microbiomes and metabolomes of three Australian ascidian species that were collected from the same geographical location at Hastings Point, NSW, Australia. To date, scientists still rely on culture-dependent methodologies in order to study the expressed secondary metabolome and its biological activity; however, this approach might miss out on unculturable 
microbial strains. Therefore, we initially determined the microbial diversity that is associated with the three ascidians based on a culture-independent approach and then compared the findings with the culturable actinomycete diversity. We further intended to provide insight into the functional role of actinomycetes in associations with ascidians and to connect the results to the production of bioactive secondary metabolites.

At the phylum level, the overall microbial diversities and distributions associated with the three Australian ascidian specimens were comparably similar. Other culture-independent ascidian microbiome studies from different geographical regions, including Asian-Pacific, South Pacific, and the Caribbean, reported similar phylum distributions, where Proteobacteria were generally reported as the most dominant phylum and also Cyanobacteria were commonly found in high abundance $[9,17,19,47-49]$. Actinobacteria were usually reported in lower numbers in these studies. However, low numbers of actinobacterial sequences could potentially be attributed to poor DNA extraction and primer biases of the V3-V4 region of the 16S rRNA gene [50]. Particularly, for Gram-positive bacteria, DNA extractions have been proven difficult and most commercial DNA extraction kits require specific cell lysis pre-treatments of the samples, which could be a reason that higher numbers of Gram-negative bacteria were reported [50].

The isolation media were targeted at mycelium forming actinomycete genera, this included Streptomyces, Micromonospora, and Nocardia, which are renowned to produce bioactive secondary metabolites. As detected in the culture-independent sequencing analysis, we successfully isolated highly diverse Streptomyces species strains from all of the ascidian samples. Micromonospora species were also obtained in high diversity and abundance from all three ascidians, while only S. rubra and P. vasculosum showed Micromonosporaceae OTUs in the culture-independent analysis. Other actinomycete resembling family members, including Gordoniaceae, Corynebacteriaceae, and Micrococcaceae colonies were also observed on isolation plates, confirming culture-independent data; however, due to time constraints they were not targeted for isolation. Streptosporangiaceae sequences were identified in S. rubra and P. vasculosum, but could only be recovered from S. rubra, which could have been affected by the choice of isolation media. Nocardia and Rhodococcus were isolated in very low numbers and displayed little diversity that might be due to their transitory characteristics within the ascidian hosts. Similarly, the chosen isolation media are commonly used for a broad range of actinomycete taxa and may not have been selective enough to culture these genera.

It has been proposed that microbial diversity of ascidians is stable and species-specific, but it also has a location-specific aspect where microorganisms are acquired from the local environment $[9,10]$. Actinomycetes are commonly found in the marine as well as the terrestrial environment. In fact, it is commonly believed that the order is of terrestrial origin and its spores have been introduced to the marine environment as wash off and then adapted to the saline conditions [51-54]. Hence, it is likely that actinomycetes are facultative symbionts, meaning that they are acquired from the environment and thus should be location-specific rather than host specific [10,55]. Nonetheless, actinomycetes still present a valuable target species for biodiscovery, especially as it is proposed that as they adapt to the marine environment, this adaptation also leads to a change in their expressed secondary metabolome [51,54,56,57]. If actinomycete associations are facultative, then actinomycete diversity of ascidians sampled at the same location and time should greatly overlap. The study on these three different ascidians collected at nearby geographic locations showed otherwise; most culturable actinomycete isolates were unique to only one ascidian. Only two cultured actinomycete OTUs were shared between the three different ascidian species; it is noteworthy that these strains were fairly abundant, pointing towards a location factor. S. rubra, which was sampled at the small rocky reef that was approximately $200 \mathrm{~m}$ offshore, had a more diverse microbial community based on Illumina sequencing data, as well as culturable actinomycete diversity when compared to the other two ascidian species. It might be possible that $S$. rubra receives more microorganisms through oceanic currents and terrestrial waters from the river mouth of Cudgara Creek that is adjacent to the sampling site (Figure 1). A. solidum and P. vasculosum were obtained from rock pools just off the mainland that provides more 
shelter from oceanic currents. Additionally, A. solidum incorporate sand internally into their tissues as they form hard rigid colony structures [58]; therefore, the low microbial diversity that is associated with $A$. solidum might be attributed to its tissue being significantly higher in sand content, allowing for less living tissue per standard volume. Since the overlap of actinomycete isolates between the different ascidian species sourced from the same physiological environment was not found to be significant, these findings support the argument that the acquisition of actinomycetes is selective. It has to be taken into account that these conclusions were established based on the actinomycetes that could be derived from the chosen isolation media and solely three nearby collected specimens and further studies would be required to confirm these conclusions.

Polyclinidae after Didemnidae is the ascidian family that has yielded most reported natural products [31]. Numerous secondary metabolites (138) from the genus Aplidium are listed in the Dictionary of Natural Products (http:/ / dnp.chemnetbase.com, accessed 30 August 2017), while only three and one natural products are listed for Symplegma and Polyclinum, respectively. These reports fit the diversity of molecular ions that are associated with three ascidians in this study. We investigated whether metabolite diversity of the three Australian ascidians was correlated with the host's microbial diversity. Our results show that the microbial diversity did not reflect the chemical diversity. S. rubra, which contained an impressive diversity of microbial associates based on culture-independent, as well as culture-dependent, approaches, had lower chemical diversity by LC-MS/MS analysis than the other two ascidian specimens. While $A$. solidum exhibited the highest metabolite diversity, low numbers of microbial sequences and culturable actinomycetes were recovered. Similarly, Tianero et al. found no correlation between microbial and chemical diversity nor biological activity, but rather specific interactions with bioactive secondary metabolite producers [9].

To gain an overview as to whether there were any secondary metabolites that can be attributed to actinomycete associates that are isolated from these ascidians, we acquired LC-MS/MS profiles of all of the ascidians and their cultured actinomycete associates. Many nodes overlapped between ascidians and actinomycetes, indicating that these compounds are potentially produced by microbial associates. Generally, ions in a molecular network are all closely related and therefore it is likely that as soon as there is one ion match between actinomycete and its host, that all compounds within this network are of microbial origin. The molecular network indicates that actinomycetes derived from Australian ascidians contain a highly diverse metabolome that in this case even exceeds that of the ascidians and targeting actinomycete associates thus proves a valuable approach for natural product drug discovery and aids to overcome the supply problem. Even more so, as bacterial cultures can be manipulated in the laboratory by using different fermentation media or using different chemical or physical elicitors to activate silent gene clusters to further induce secondary metabolite diversity [59].

In this study, we presented a rich taxonomic as well as metabolite diversity of actinomycetes that are associated with ascidians that both appear to be species specific. The geographic and taxonomic scope was however limited to only three ascidian species from one habitat, therefore we regard this work as a small contribution to the bigger picture. Extended knowledge of the diversity and distribution of actinomycetes in the marine environment will contribute further to a deeper understanding of their ecological function and will aid towards the design of improved biodiscovery strategies. The next steps in this work will be to identify new secondary metabolites that could not be assigned from the molecular network analysis and thus maximise the immense potential of actinomycetes for natural product drug discovery.

\section{Conclusions}

This study has shown that the ascidians, S. rubra, A. solidum, and P. vasculosum, harbour a range of diverse actinomycetes, which we successfully identified with culture independent methods and were subsequently isolated using culture dependent techniques. The actinomycete metabolomes, specifically for Streptomyces and Micromonospora isolates, were highly diverse and thus present a good target for natural product drug discovery. 
Supplementary Materials: The following are available online at www.mdpi.com/1424-2818/9/4/53/s1, Table S1: Ascidian-associated actinomycete library details, Table S2: Full molecular networking ion nodes list.

Acknowledgments: The authors would like to thank Tanja Grkovic for providing valuable feedback to the manuscript. Nathan Caromel and Johan Gustafson were of tremendous help in collecting the ascidian samples. Larissa Buedenbender thanks Candice Brinkmann for the much valued advice in laboratory practices and recognizes Leesa Habener's assistance in constructing the phylogenetic trees. The authors gratefully acknowledge the support of the Griffith University eResearch Services Team and the use of the High-Performance Computing Cluster "Gowonda" for QIIME analysis. Larissa Buedenbender acknowledges the provision of an Australian Postgraduate Award and Griffith University International Postgraduate Research Scholarship. The research was funded by the Griffith School of Environment.

Author Contributions: Larissa Buedenbender and D. İpek Kurtböke designed and conceived the experiments; Larissa Buedenbender organised the sampling event, performed the experiments, conducted molecular work, analysed the microbiome, as well as microbiological and chemical data; D. İpek Kurtböke and Anthony R. Carroll contributed reagents and analysis tools; Merrick Ekins identified the ascidian specimens to species level. Larissa Buedenbender wrote the manuscript. Anthony R. Carroll and D. İpek Kurtböke supervised Larissa Buedenbender's Ph.D. project and provided feedback to the manuscript.

Conflicts of Interest: The authors declare no conflict of interest.

\section{References}

1. Paul, V.J.; Lindquist, N.; Fenical, W. Chemical defenses of the tropical ascidian Atapozoa sp. and its nudibranch predators Nembrotha spp. Mar. Ecol. Prog. Ser. 1990, 59, 109-118. [CrossRef]

2. Lindquist, N.; Hay, M.E.; Fenical, W. Defense of ascidians and their conspicuous larvae: Adult vs. Larval chemical defenses. Ecol. Monogr. 1992, 62, 547-568. [CrossRef]

3. Joullié, M.M.; Leonard, M.S.; Portonovo, P.; Liang, B.; Ding, X.; La Clair, J.J. Chemical defense in ascidians of the didemnidae family. J. Bioconj. Chem. 2003, 14, 30-37. [CrossRef] [PubMed]

4. Meyer, C.A. The Global Marine Pharmaceuticals Pipeline. Available online: http:/ / marinepharmacology. midwestern.edu/clinPipeline.htm (accessed on 4 April 2017).

5. Schmidt, E.W.; Nelson, J.T.; Rasko, D.A.; Sudek, S.; Eisen, J.A.; Haygood, M.G.; Ravel, J. Patellamide A and C biosynthesis by a microcin-like pathway in prochloron didemni, the cyanobacterial symbiont of lissoclinum patella. Proc. Natl. Acad. Sci. USA 2005, 102, 7315-7320. [CrossRef] [PubMed]

6. Schmidt, E.W. The secret to a successful relationship: Lasting chemistry between ascidians and their symbiotic bacteria. Invertebr. Biol. 2014, 134, 88-102. [CrossRef] [PubMed]

7. Leal, M.C.; Sheridan, C.; Osinga, R.; Dionísio, G.; Rocha, R.J.M.; Silva, B.; Rosa, R.; Calado, R. Marine microorganism-invertebrate assemblages: Perspectives to solve the "supply problem" in the initial steps of drug discovery. Mar. Drugs 2014, 12, 3929-3952. [CrossRef] [PubMed]

8. Radjasa, O.K.; Vaske, Y.M.; Navarro, G.; Vervoort, H.C.; Tenney, K.; Linington, R.G.; Crews, P. Highlights of marine invertebrate-derived biosynthetic products: Their biomedical potential and possible production by microbial associants. Bioorg. Med. Chem. 2011, 19, 6658-6674. [CrossRef] [PubMed]

9. Tianero, M.D.B.; Kwan, J.C.; Wyche, T.P.; Presson, A.P.; Koch, M.; Barrows, L.R.; Bugni, T.S.; Schmidt, E.W. Species specificity of symbiosis and secondary metabolism in ascidians. ISME J. 2014, 9, 615-628. [CrossRef] [PubMed]

10. Erwin, P.M.; Pineda, M.C.; Webster, N.; Turon, X.; Lopez-Legentil, S. Down under the tunic: Bacterial biodiversity hotspots and widespread ammonia-oxidizing archaea in coral reef ascidians. ISME J. 2014, 8, 575-588. [CrossRef] [PubMed]

11. Jensen, P.R.; Fenical, W. Strategies for the discovery of secondary metabolites from marine bacteria: Ecological perspectives. Annu. Rev. Microbiol. 1994, 48, 559-584. [CrossRef] [PubMed]

12. Kwan, J.C.; Schmidt, E.W. Bacterial endosymbiosis in a chordate host: Long-term co-evolution and conservation of secondary metabolism. PLoS ONE 2013, 8, e80822. [CrossRef] [PubMed]

13. Kwan, J.C.; Tianero, M.D.B.; Donia, M.S.; Wyche, T.P.; Bugni, T.S.; Schmidt, E.W. Host control of symbiont natural product chemistry in cryptic populations of the tunicate lissoclinum patella. PLoS ONE 2014, 9, e95850. [CrossRef] [PubMed]

14. Kremer, B.; Pardy, R.; Lewin, R. Carbon fixation and photosynthates of prochloron, a green alga symbiotic with an ascidian, lissoclinum patella. Phycologia 1982, 21, 258-263. [CrossRef] 
15. Koike, I.; Suzuki, T. Nutritional diversity of symbiotic ascidians in a fijian seagrass meadow. Ecol. Res. 1996, 11, 381-386. [CrossRef]

16. Münchhoff, J.; Hirose, E.; Maruyama, T.; Sunairi, M.; Burns, B.P.; Neilan, B.A. Host specificity and phylogeography of the prochlorophyte Prochloron sp., an obligate symbiont in didemnid ascidians. Environ. Microbiol. 2007, 9, 890-899. [CrossRef] [PubMed]

17. Donia, M.S.; Fricke, W.F.; Partensky, F.; Cox, J.; Elshahawi, S.I.; White, J.R.; Phillippy, A.M.; Schatz, M.C.; Piel, J.; Haygood, M.G. Complex microbiome underlying secondary and primary metabolism in the tunicate-prochloron symbiosis. Proc. Natl. Acad. Sci. USA 2011, 108, E1423-E1432. [CrossRef] [PubMed]

18. Donia, M.S.; Fricke, W.F.; Ravel, J.; Schmidt, E.W. Variation in tropical reef symbiont metagenomes defined by secondary metabolism. PLoS ONE 2011, 6, 17897. [CrossRef] [PubMed]

19. Steinert, G.; Taylor, M.W.; Schupp, P.J. Diversity of actinobacteria associated with the marine ascidian eudistoma toealensis. Mar. Biotechnol. 2015, 17, 377-385. [CrossRef] [PubMed]

20. Chen, L.; Fu, C.; Wang, G. Microbial diversity associated with ascidians: A review of research methods and application. Symbiosis 2016, 71, 19-26. [CrossRef]

21. Jimenez, P.C.; Ferreira, E.G.; Araújo, L.A.; Guimarães, L.A.; Sousa, T.S.; Pessoa, O.D.L.; Lotufo, T.M.; Costa-Lotufo, L.V. Cytotoxicity of actinomycetes associated with the ascidian eudistoma vannamei (millar, 1977), endemic of northeastern coast of brazil/citotoxicidad de actinomicetos asociada a la ascidia eudistoma vannamei (millar, 1977), endémica de la costa noreste de Brasil. Lat. Am. J. Aquat. Res. 2013, 41, 335.

22. List of Prokaryotic Names with Standing in Nomenclature. Available online: http://www.bacterio.net/ actinomycetales.html (accessed on 16 November 2017).

23. Kurtböke, D.I. Ecology and habitat disctribution of actinobacteria. In Biology and Biotechnology of Actinobacteria; Wink, J., Hamedi, J., Eds.; Springer: Berlin, Germany, 2017; pp. 123-149.

24. He, H.; Ding, W.-D.; Bernan, V.S.; Richardson, A.D.; Ireland, C.M.; Greenstein, M.; Ellestad, G.A.; Carter, G.T. Lomaiviticins $a$ and $b$, potent antitumor antibiotics from micromonospora lomaivitiensis. J. Am. Chem. Soc. 2001, 123, 5362-5363. [CrossRef] [PubMed]

25. Li, A.; Piel, J. A gene cluster from a marine streptomyces encoding the biosynthesis of the aromatic spiroketal polyketide griseorhodin A. Chem. Biol. 2002, 9, 1017-1026. [CrossRef]

26. Hayakawa, Y.; Shirasaki, S.; Kawasaki, T.; Matsuo, Y.; Adachi, K.; Shizuri, Y. Structures of new cytotoxic antibiotics, piericidins C7 and C8. J. Antibiot. 2007, 60, 201. [CrossRef] [PubMed]

27. Wyche, T.P.; Hou, Y.; Vazquez-Rivera, E.; Braun, D.; Bugni, T.S. Peptidolipins bf, antibacterial lipopeptides from an ascidian-derived Nocardia sp. J. Nat. Prod. 2012, 75, 735. [CrossRef] [PubMed]

28. Zhang, Y.; Adnani, N.; Braun, D.R.; Ellis, G.A.; Barns, K.J.; Parker-Nance, S.; Guzei, I.A.; Bugni, T.S. Micromonohalimanes A and B: Antibacterial halimane-type diterpenoids from a marine micromonospora species. J. Nat. Prod. 2016, 79, 2968-2972. [CrossRef] [PubMed]

29. Horton, P.; Longley, R.; McConnell, O.; Ballas, L. Staurosporine aglycone (K252-c) and arcyriaflavin a from the marine ascidian, Eudistoma sp. Cell. Mol. Life Sci. 1994, 50, 843-845. [CrossRef]

30. Andréo, M.A.; Jimenez, P.C.; Siebra, J.B.; Costa-Lotufo, L.V.; Vessecchi, R.; Niehues, M.; Lopes, J.L.; Lopes, N.P. Systematic UPLC-ESI-MS/MS study on the occurrence of staurosporine and derivatives in associated marine microorganisms from eudistoma vannamei. J. Braz. Chem. Soc. 2012, 23, 335-343. [CrossRef]

31. Leal, M.C.; Puga, J.; Serodio, J.; Gomes, N.C.M.; Calado, R. Trends in the Discovery of New Marine Natural Products from Invertebrates over the Last Two Decades-Where and What Are We Bioprospecting? PLoS ONE 2012, 7, e30580. [CrossRef] [PubMed]

32. Sherman, C.D.; Hunt, A.; Ayre, D.J. Is life history a barrier to dispersal? Contrasting patterns of genetic differentiation along an oceanographically complex coast. Biol. J. Linn. Soc. 2008, 95, 106-116. [CrossRef]

33. Hastings Point. Microsoft 2017. Available online: www.bing.com/maps (accessed on 10 August 2017).

34. Bolger, A.M.; Lohse, M.; Usadel, B. Trimmomatic: A flexible trimmer for illumina sequence data. Bioinformatics 2014, 30, 2114-2120. [CrossRef] [PubMed]

35. Masella, A.P.; Bartram, A.K.; Truszkowski, J.M.; Brown, D.G.; Neufeld, J.D. Pandaseq: Paired-end assembler for illumina sequences. BMC Bioinform. 2012, 13, 31. [CrossRef] [PubMed]

36. Caporaso, J.G.; Kuczynski, J.; Stombaugh, J.; Bittinger, K.; Bushman, F.D.; Costello, E.K.; Fierer, N.; Pena, A.G.; Goodrich, J.K.; Gordon, J.I. Qiime allows analysis of high-throughput community sequencing data. Nat. Methods 2010, 7, 335-336. [CrossRef] [PubMed] 
37. Küster, E.; Williams, S. Selection of media for isolation of streptomycetes. Nature 1964, 202, 928-929. [CrossRef]

38. English, A.L.; Boufridi, A.; Quinn, R.; Kurtböke, D. Evaluation of fermentation conditions triggering increased antibacterial activity from a near-shore marine intertidal environment-associated streptomyces species. Synth. Syst. Biotechnol. 2017, 2, 28-38. [CrossRef] [PubMed]

39. Atlas, R.M. Handbook of Microbiological Media; CRC Press: Boca Raton, FL, USA, 2010.

40. Williams, S.T.; Wellington, E.M.H. Actinomycetes. In Methods of Soil Analysis. Part 2. Chemical and Microbiological Properties; Page, A.L., Ed.; American Society of Agronomy, Soil Science Society of America: Madison, WI, USA, 1982; pp. 969-987.

41. Wellington, E. Preservation of actinomycete inoculum in frozen glycerol. Microbios. Lett. 1979, 6, 151-157.

42. Edgar, R.C. Muscle: Multiple sequence alignment with high accuracy and high throughput. Nucleic Acids Res. 2004, 32, 1792-1797. [CrossRef] [PubMed]

43. Wang, M.; Carver, J.J.; Phelan, V.V.; Sanchez, L.M.; Garg, N.; Peng, Y.; Nguyen, D.D.; Watrous, J.; Kapono, C.A.; Luzzatto-Knaan, T. Sharing and community curation of mass spectrometry data with global natural products social molecular networking. Nat. Biotechnol. 2016, 34, 828-837. [CrossRef] [PubMed]

44. Takada, K.; Ninomiya, A.; Naruse, M.; Sun, Y.; Miyazaki, M.; Nogi, Y.; Okada, S.; Matsunaga, S. Surugamides a-e, cyclic octapeptides with four d-amino acid residues, from a marine Streptomyces sp.: Lc-ms-aided inspection of partial hydrolysates for the distinction of d-and l-amino acid residues in the sequence. J. Org. Chem. 2013, 78, 6746-6750. [CrossRef] [PubMed]

45. Aoyagi, T.; Takeuchi, T.; Matsuzaki, A.; Kawamura, K.; Kondo, S.; Hamada, M.; Maeda, K.; Umezawa, H. Leupeptins, new protease inhibitors from actinomycetes. J. Antibiot. 1969, 22, 283-286. [CrossRef] [PubMed]

46. McBrien, K.D.; Berry, R.L.; Lowe, S.E.; Neddermann, K.M.; Bursuker, I.; Huang, S.; Klohr, S.E.; Leet, J.E. Rakicidins, new cytotoxic lipopeptides from Micromonospora sp. Fermentation, isolation and characterization. J. Antibiot. 1995, 48, 1446-1452. [CrossRef] [PubMed]

47. Martínez-García, M.; Díaz-Valdés, M.; Wanner, G.; Ramos-Esplá, A.; Antón, J. Microbial community associated with the colonial ascidian cystodytes dellechiajei. Environ. Microbiol. 2007, 9, 521-534. [CrossRef] [PubMed]

48. Tait, E.; Carman, M.; Sievert, S.M. Phylogenetic diversity of bacteria associated with ascidians in EEL pond (Woods Hole, Massachusetts, USA). J. Exp. Mar. Biol. Ecol. 2007, 342, 138-146. [CrossRef]

49. Behrendt, L.; Larkum, A.W.; Trampe, E.; Norman, A.; Sørensen, S.J.; Kühl, M. Microbial diversity of biofilm communities in microniches associated with the didemnid ascidian lissoclinum patella. ISME J. 2012, 6, 1222-1237. [CrossRef] [PubMed]

50. Morgan, J.L.; Darling, A.E.; Eisen, J.A. Metagenomic sequencing of an in vitro-simulated microbial community. PLoS ONE 2010, 5, e10209. [CrossRef] [PubMed]

51. Sponga, F.; Cavaletti, L.; Lazzarini, A.; Borghi, A.; Ciciliato, I.; Losi, D.; Marinelli, F. Biodiversity and potentials of marine-derived microorganisms. J. Biotechnol. 1999, 70, 65-69. [CrossRef]

52. Jensen, P.R.; Gontang, E.; Mafnas, C.; Mincer, T.J.; Fenical, W. Culturable marine actinomycete diversity from tropical pacific ocean sediments. Environ. Microbiol. 2005, 7, 1039-1048. [CrossRef] [PubMed]

53. Penn, K.; Jensen, P.R. Comparative genomics reveals evidence of marine adaptation in salinispora species. BMC Genom. 2012, 13, 86. [CrossRef] [PubMed]

54. Kurtböke, D.İ.; Grkovic, T.; Quinn, R.J. Marine actinomycetes in biodiscovery. In Springer Handbook of Marine Biotechnology; Kim, S.-K., Ed.; Springer: Berlin, Germany, 2015; pp. 663-676.

55. Sunagawa, S.; Woodley, C.M.; Medina, M. Threatened corals provide underexplored microbial habitats. PLoS ONE 2010, 5, e9554. [CrossRef] [PubMed]

56. Okami, Y.; Okazaki, T.; Kitahara, T.; Umezawa, H. Studies on marine microorganisms. V. J. Antibiot. 1976, 29, 1019-1025. [CrossRef] [PubMed]

57. Imada, C.; Koseki, N.; Kamata, M.; Kobayashi, T.; Hamada-Sato, N. Isolation and characterization of antibacterial substances produced by marine actinomycetes in the presence of seawater. Actinomycetologica 2007, 21, 27-31. [CrossRef] 
58. Kott, P. Ascidiacea (Tunicata) from deep waters of the continental shelf of Western Australia. J. Nat. Hist. 2008, 42, 1103-1217. [CrossRef]

59. Scherlach, K.; Hertweck, C. Triggering cryptic natural product biosynthesis in microorganisms. Org. Biomol. Chem. 2009, 7, 1753-1760. [CrossRef] [PubMed] 